\title{
Dynamic 3D Ultrasound and MR Image Registration of the Beating Heart
}

\author{
Xishi Huang ${ }^{1,6}$, Nicholas A. Hill ${ }^{2,6}$, Jing Ren $^{3}$, Gerard Guiraudon ${ }^{4,6}$, \\ Derek Boughner, ${ }^{5,6}$ and Terry M. Peters ${ }^{1,2,6}$ \\ ${ }^{1}$ Department of Biomedical Engineering \\ ${ }^{2}$ Department of Medical Biophysics \\ ${ }^{3}$ Department of Electrical and Computer Engineering, \\ The University of Western Ontario \\ ${ }^{4}$ Canadian Surgical Technology and Advanced Robotics (CSTAR) \\ ${ }^{5}$ London Health Sciences Centre \\ ${ }^{6}$ Imaging Research Labs, Robarts Research Institute, London, \\ ON, Canada N6A 5K8 \\ \{xhuang, nhill, tpeters\}@imaging.robarts.ca \\ \{jren2, gguiraud\} @uwo.ca \\ derek. boughner@lhsc.on.ca
}

\begin{abstract}
Real-time three-dimensional ultrasound (RT3D US) is an ideal imaging modality for the diagnosis of cardiac disease. RT3D US is a flexible, inexpensive, non-invasive tool that provides important diagnostic information related to cardiac function. Unfortunately, RT3D US suffers from inherent shortcomings, such as low signal-to-noise ratio and limited field of view, producing images that are difficult to interpret. Multi-modal dynamic cardiac image registration is a well-recognized approach that compensates for these deficiencies while retaining the advantages of RT3D US imaging. The clinical application of multi-modal image registration methods is difficult, and there are a number of implementation issues to be resolved. In this work, we present a method for the rapid registration of RT3D US images of the beating heart to high-resolution magnetic resonance (MR) images. This method was validated using a volunteer image set. Validation results demonstrate that this approach can achieve rapid registration of images of the beating heart with fiducial landmark and registration errors of $1.25 \pm 0.63$ and $1.76 \mathrm{~mm}$ respectively. This technique can potentially be used to improve the diagnosis of cardiac disease by augmenting RT3D US images with high-resolution MR images and to facilitate intra-operative image fusion for minimally invasive cardio-thoracic surgical navigation.
\end{abstract}

\section{Introduction}

Cardiovascular disease is the most frequent cause of death by disease in North America and accounts for the death of more Canadians than any other disease. Early diagnosis of heart failure is essential for successfully addressing the underlying diseases and/or causes, and the prevention of further myocardial dysfunction and clinical deterioration. 
The ability of MRI to be employed in a dynamic mode allows cardiologists to acquire high quality images of cardiac anatomy that assist diagnosis, yet it is an expensive procedure that may not yield all of the relevant diagnostic information. Echocardiography, also used to diagnose cardiac disease, is a flexible, inexpensive, non-invasive tool. However, the cardiac images produced are of lower quality and the small field of view (FOV) makes it difficult for the cardiologist to mentally place abnormal cardiac structures and congenital defects in the proper clinical context, which increases the risk of misdiagnosis. While both real time echocardiography and dynamic MRI are routinely used in the diagnosis of cardiac disease, there is no mechanism to easily integrate information from both image sets in order to take maximum advantage of both modalities. Techniques currently used to examine the heart provide valuable information, but do not represent the complete picture of cardiac health. It is therefore important for the cardiologist to dynamically relate the images generated from US studies to dynamic MR images from the same patient. We believe that ability to correlate real time echocardiography images with previously acquired dynamic 3D MR images would be a significant contribution to the diagnosis of cardiovascular abnormalities and to interventional and minimally-invasive cardiac surgery.

While existing literature outlines procedures and methods for multi-modal image registration [1, 2, 3], these approaches have mainly been used in neurosurgical applications [4, 5] and abdominal interventions [6]. These methods show promising results, however these techniques are insufficient to meet the demands of real time cardiac image registration. To account for the periodic motion of the beating heart, image registration must be performed very rapidly. The lower quality of the US images of the heart makes the realization of a fast, robust US-MR image registration technique difficult.

In this paper, we present a method for the rapid registration of RT3D US images with dynamic 3D MR images. This method integrates electrocardiogram (ECG) signals and a spatial tracking system with a commercially available US machine. Compared to the existing methods for fusing RT3D US with dynamic 3D MR images, our technique is the first to simultaneously address the issues of image acquisition, image processing timing constraints, and the motion of the beating heart.

\section{Methods}

In medical image registration one approach for modeling the beating heart is to represent the heart as a deformable model [7]. A major concern relating to this approach for real time 3D US-MR image registration is the associated computation time. For the method to operate in real time, the entire process (image acquisition, processing and visualization) must be completed at least 20 times per second. Accounting for time lag due to image acquisition and visualization, for real time image integration to be possible, image registration must be completed in $20-50 \mathrm{~ms}$. If a deformable model is used, then the integration process requires substantially more computation time, because additional registration parameters need to be adjusted and optimized [8]. Therefore, the use of a deformable model to represent the heart is not a suitable choice in this case. To meet these strict time constraints we propose a registration method that employs a rigid-body transformation between US and MR images. 


\subsection{Rigid-Body Representation}

During cardiac diagnosis and surgery planning the heart remains relatively fixed with respect to the thoracic cage between dynamic 3D MRI and RT3D US examinations. Breath-holding is employed during both MRI and US acquisitions to ensure that organ motion is due solely to the beating heart, and not a combination of the beating heart and patient respiration. Although the shape and size of the heart differs at different phases in the cardiac cycle, it is reasonable to assume that the overall pattern of shape and size variation does not change beat-to-beat. This assumption is especially true in the case of diagnosis where there is no significant change in heart rate or blood pressure. We further observe that if we subdivide the cardiac cycle into discrete cardiac phases, the US and MR images acquired at the same moment in the cardiac cycle represent the same physical heart features. Given these observations, it is straightforward to represent the registration for each pair of the US and MR frames of the same cardiac phase with a rigid-body transformation.

Furthermore, if we consider any two pairs of US and MR images in different phases, the non-rigid transformation between the two MRI frames is the same as that for the two US frames, because they represent the same physical heart deformation between the two cardiac phases. Aligning any pair of MRI and US frames automatically aligns the other sets of frames.

These observations imply that a single rigid-body transformation between the MR images and US images can be used to rapidly register the image sets.

\subsection{Registration Method}

To register RT3D US images with 4D MR images we track the US probe using a Polaris optical tracking system (OTS) (NDI, Waterloo, Canada) while simultaneously recording ECG signals. One major advantage of using US imaging systems lies in the flexibility of image acquisition. During a cardiac examination, the operator has the freedom to position/orientate the US probe in any manner to obtain the necessary views of the heart. Since the position and orientation of US probe is continuously tracked with the Polaris OTS, the tracking information can be used to register the US images to the world coordinate system (WCS).

ECG signals are employed to temporally align the US and MRI frames. Dynamic 3D MRI frames are often acquired with a fixed sampling rate, whereas the sampling rates for US systems vary with any on-the-fly adjustments made to the FOV by the operator. This difference in sampling rates implies that the US and MRI frames will not in general be temporally synchronized. To overcome this difficulty, we utilize ECG signals to phase-stamp the US images with timing information derived from the ECG signals. The MR images are temporally interpolated, and using this phase data, US and MR images with coincident cardiac phases are identified. The US images are transformed into the WCS and the US and MR images are registered together. Recognizing that there will be sampling errors in the ECG signal, the tracking system, and variations in the heart rate between MRI and US acquisitions, it is necessary to perform a final 'fine-tuning' of the registration to ensure optimal spatial and temporal alignment. 
In order to fine-tune the registration result, the transformation matrix generated by the registration procedure is used as the starting position/orientation for a mutual information (MI) registration method [9], which further optimizes the registration with respect to seven parameters: translations $(x, y, z)$, rotations $\left(\theta_{x}, \theta_{y}, \theta_{z}\right)$ and time $(t)$.

\subsection{Registration Procedure}

Based on the above considerations and the analysis of cardiac diagnostic and planning problems, we outline the following procedures for real time image registration. Our approach involves two steps: 1) a pre-registration step and 2) a real-time registration step (Figure 1). The steps for the registration procedure are as follows:

Step 1. Pre-registration before procedure (diagnosis/planning):

Pre-operative

1. acquire dynamic 3D MR images (gradient echo T1-weighted imaging sequence with a voxel size of $1.5 \mathrm{~mm}^{3}$ [10]) at different phases over one cardiac cycle

Immediately prior to procedure

2. acquire a 3D US image (denoted $U S_{l}$ ) recording the US probe's tracking information $\left(T_{W C S \leftarrow U S_{1}}\right.$ ) and the ECG signal at some cardiac phase

3. the MR images are temporally interpolated and based on the ECG information $\left(\hat{t}_{1}\right)$ the MR image with the closest cardiac phase is identified (denoted $m r_{l}$ )

4. the two image sets are manually registered together, generating a temporary transformation matrix, $T_{m r_{1} \leftarrow U S_{1}}$

5. using $T_{m r_{1} \leftarrow U S_{1}}$ as the starting point, the two image sets are registered together using a MI registration method, optimizing the positional, rotational and temporal variables $\left(x, y, z, \theta_{x}, \theta_{y}, \theta_{z}, t\right)$ to obtain $T_{M R_{1} \leftarrow U S_{1}}$. We use the notation $M R_{l}$ to denote the optimal aligned MR image corresponding to $U S_{I}$

6. finally, using the following relation, the pre-registration transformation $T_{M R \leftarrow W C S}$ is calculated

$$
T_{M R \leftarrow W C S}=T_{M R_{1} \leftarrow U S_{1}}\left(T_{W C S \leftarrow U S_{1}}\right)^{-1}
$$

Step 2: Real-time registration during procedure:

1. acquire an intra-operative RT3D US image (denoted $U S_{2}$ ), recording the probe tracking information $\left(T_{W C S \leftarrow U S_{2}}\right)$ and ECG signal

2. use the ECG signal to estimate the cardiac phase, $\hat{t}_{2}$, corresponding to $U S_{2}$

3. derive a 'near-optimal' transformation, $T_{m r_{2} \leftarrow U S_{2}}$, using the following equation

$$
T_{m r_{2} \leftarrow U S_{2}}=T_{M R \leftarrow W C S} T_{W C S \leftarrow U S_{2}}
$$

where $T_{M R \leftarrow W C S}$ is the initial position/orientation estimated from the pre-registration procedure, and $T_{W C S \leftarrow U S_{2}}$ is the US to WCS calibration transformation for $U S_{2}$ and $m r_{2}$ denotes the MR image interpolated based on the ECG information $\left(\hat{t}_{2}\right)$ 
4. using $\hat{t}_{2}$ as the cardiac phase and $T_{m r_{2} \leftarrow U S_{2}}$ as the starting position/orientation, register the two image sets together using a MI registration method and generate the final transformation, $T_{M R_{2} \leftarrow U S_{2}}$, where $M R_{2}$ denotes the optimal interpolated MR image corresponding to $U S_{2}$.

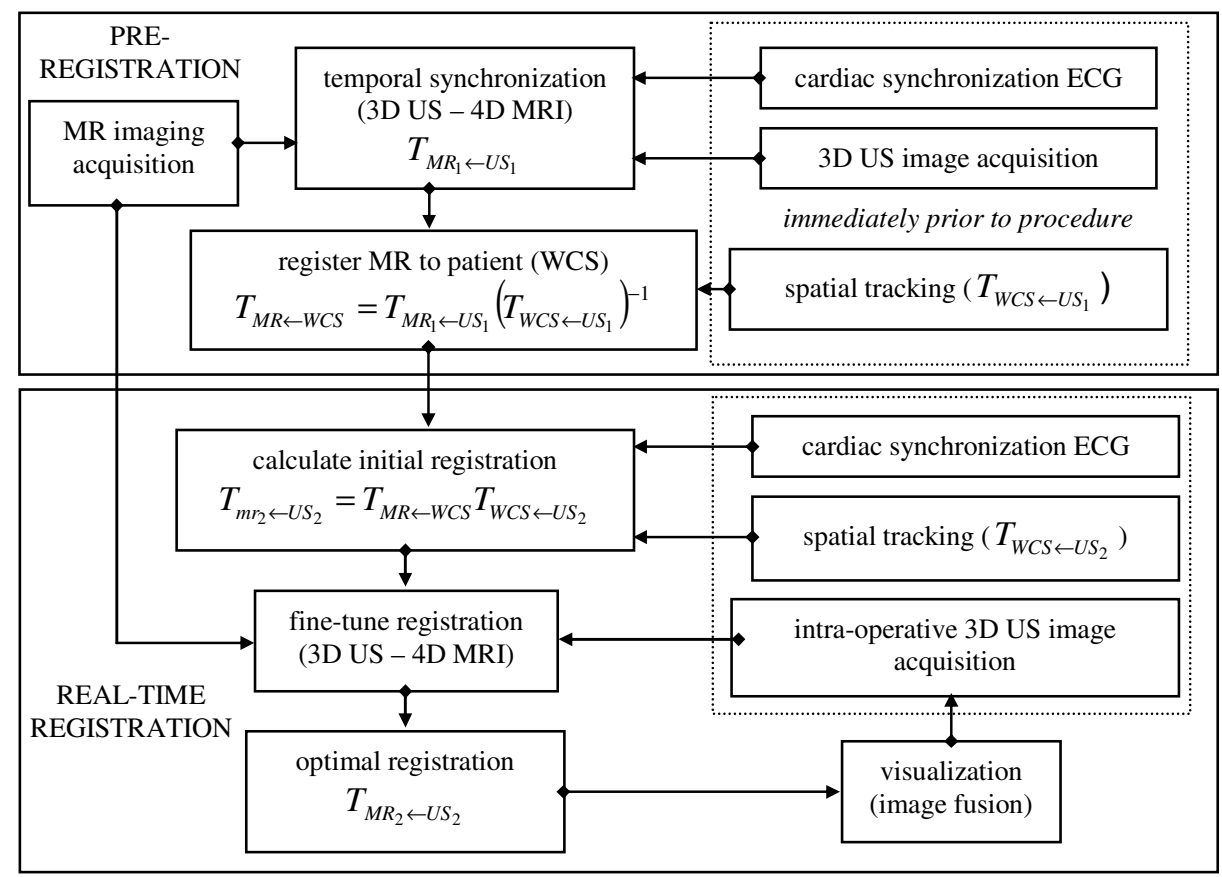

Fig. 1. The proposed method is a two-step registration procedure. The first step, preregistration, involves registering pre-operative dynamic 3D MR images to RT3D US images at an arbitrary point in the cardiac cycle. This step is performed while the patient is on the OR/examination table. The second step, real-time registration, is performed during the procedure and involves acquiring RT3D US images augmented with both ECG signals and spatial tracking information. A series of these 'augmented' RT3D US images are continuously acquired and registered with the pre-operative dynamic 3D MR images to provide accurate, real time image registration.

\section{Experimental Results}

In this experiment, 20 dynamic 3D MR images of one cardiac cycle, acquired on a 1.5T GE CVi scanner (GE Medical systems, Milwaukee) and 14 RT3D US images, acquired on a Phillips SONOS 7500 real time US machine, from the same volunteer were registered together. The image sets were temporally aligned, and then spatially registered using a MI registration algorithm. This method was able to achieve image registration within 1 second. 
The registration was visually satisfactory in all image pairs (see Figure 2), but it is not a trivial task to perform a quantitative validation, since the ground truth is unknown. We discuss below two methods used to evaluate the registration accuracy: 1) a landmark-based method, and 2) an average transformation method.

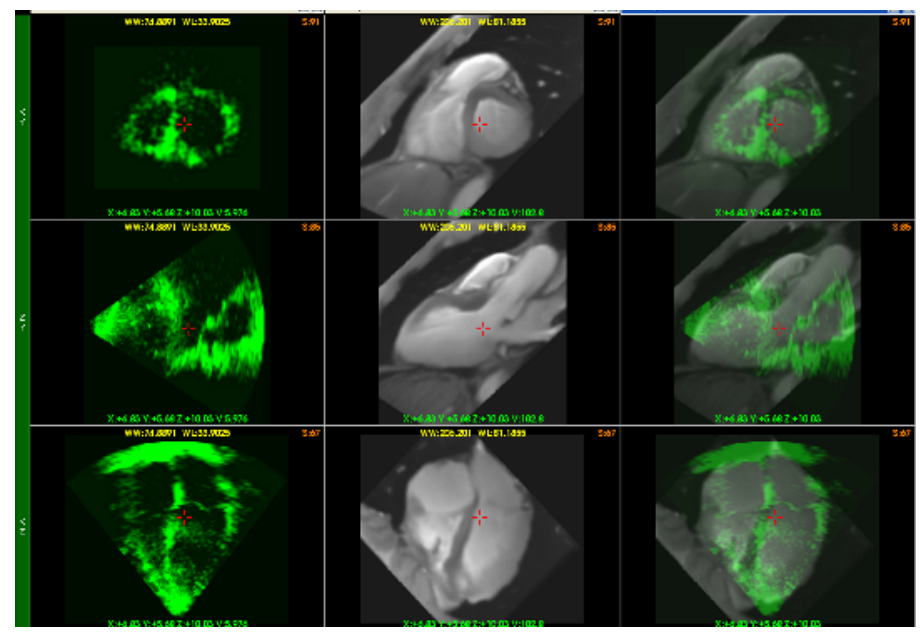

Fig. 2. Registration between RT3D US and dynamic 3D MR images. (a) orthogonal slices of the US volume of the beating heart; (b) the MRI volume of the beating heart; (c) the overlay of the two image sets after registration.

\subsection{Landmark Based Validation}

Anatomical landmarks within the heart were used to evaluate the registration accuracy. Five such landmarks, the mitral annular septal site (MASS), mitral valve (MV), anterior tricuspid valve (ATV), septal tricuspid valve (STV) and the coronary sinus (CS), were identified in both the US and MR images by six observers and the landmark (or fiducial) localization error (FLE) and fiducial registration error (FRE) were determined. The FLE is defined as the error in locating the landmarks (i.e. the distance of the localized landmark from the "forever unknown" actual landmark location) [11] and is approximated by the average of the landmark locations for the six observers. The FRE is defined as the root mean square (RMS) distance between landmarks in the US image after registration and the corresponding homologous landmarks in the MR image. In this experiment the FLE and the FRE were $1.25 \pm$ 0.63 and $1.76 \mathrm{~mm}$ respectively.

\subsection{Average Transformation Based Validation}

Since the heart beats periodically there should be little variation between the resultant registration transformations of all cardiac phases. Using this assumption it is reasonable to approximate the average transformation over all cardiac phases as the "ground truth" transformation. We evaluate the registration accuracy by using the 
average distance error, which is defined as the average of displacement error at the eight vertices of a hypothetical cube centered with the bounding box of a data volume [8]. The side of the cube is $100 \mathrm{~mm}$. We compute the average distance error from the average transformation for each registration of the paired images at the same cardiac phase. Compared to the average transformation of all cardiac phases, the average distance error is $0.86 \pm 0.40 \mathrm{~mm}$ (mean $\pm \mathrm{SD}$ ). While this result is smaller than the values represented in the landmark-based method, it is nevertheless reasonable considering the FLE previously reported.

\section{Discussion and Conclusion}

In this paper we presented a method for the rapid registration of RT3D US and dynamic 3D MR images of the beating heart. This technique will improve the ease and accuracy of cardiac disease diagnosis, as well as aid in surgical planning and guidance.

We employed image data of a volunteer's beating heart to validate the proposed method with encouraging results. In the future we plan to investigate more effective approaches to preprocessing US images and develop a more robust registration method to improve the registration accuracy and speed.

This method can also be employed to register real-time dynamic 2D US images with dynamic 3D pre-operative CT/MR images and other multi-modal dynamic images, and has the potential to be used in other clinical applications such as liver and lung surgeries, where the organs are subject to approximately periodic respiratory motion.

This method was validated using volunteer data to yield a registration accuracy of $1.76 \mathrm{~mm}$. This method will provide real-time high quality image guidance for cardiac disease diagnosis and surgical planning by improving interpretation of images of the beating heart. We also expect this work to lead to the development of a novel cardiac diagnostic US device that can output real-time high quality cardiac images, fused with high-resolution anatomical information. This device will retain all the merits of conventional US system, and will also have applicability for the guidance of intra-cardiac interventions by improving interpretation of images acquired from various cardiac US modalities (trans-thoracic, trans-esophageal and intra-cardiac echo techniques).

\section{Acknowledgements}

The authors thank Dr. Xiaping Yuan for her help in data acquisition and image processing. We also acknowledge the financial support from the Canadian Institute of Health Research (CIHR), Natural Sciences and Engineering Research Council of Canada (NSERC), the Ontario Research \& Development Challenge Fund (ORDCF), the Canada Foundation for Innovation (CFI), and the Ontario Innovation Trust (OIT).

\section{References}

1. Makela, T., Clarysse, P., Sipila, O., Pauna, N., Pham, Q.C., Katila, T., Magnin, I.E., A review of cardiac image registration methods, IEEE trans. on Med. Imag., 21(9):10111021, 2002. 
2. Hill, D.L.G., Batchelor, P.G., Holden, M., Hawkes, D.J., Medical image registration, Phys. Med. Biol., 46:R1-R45, 2001.

3. Maintz, J.B., Viergever, M.A., A survey of medical image registration, Med. Image Anal., 2: 1-36, 1998.

4. Gobbi, D.G., Comeau, R.M., Peters, T.M., Ultrasound/MRI overlay with image warping for neurosurgery. MICCAI 2000, LNCS 1935, 106-114, 2000.

5. Lindseth, F., Kaspersen, J.H., Ommedal, S., Langø, T., Unsgaard, G., Hernes, T.A.N., Multimodal image fusion in ultrasound-based neuronavigation: improving overview and interpretation by integrating preoperative MRI with intraoperative 3D ultrasound, submitted to Computer Aided Surgery, 2002.

6. Kaspersen, J.H., Sjølie, E., Wesche, J., Asland, J., Lundbom, J., Odegard, A., Lindseth, F., Three-dimensional ultrasound-based navigation combined with preoperative CT during abdominal interventions: a feasibility study, Cardiovasc Intervent Radiol, 26:347-356, 2003.

7. Wierzbicki M., Drangova M., Guiraudon G., Peters T., Validation of dynamic heart models obtained using non-linear registration for virtual reality training, planning, and guidance of minimally invasive cardiac surgeries, Medical Image Analysis 8:387-401, 2004.

8. Shekhar, R., Zagrodsky, V., Mutual Information-based rigid and nonrigid registration of ultrasound volumes, IEEE Trans. on Medical Imaging, 21(1):9-22, 2002.

9. Mattes, D., Haynor, D.R., Vesselle, H., Lewellen, T.K., Eubank, W., PET-CT image registration in the chest using free-form deformations, IEEE Transactions on Medical Imaging, 22(1): 120 - 128, 2003.

10. Moore, J., Drangova, M., Wierzbicki, M., Barron, J., Peters, T., A high resolution dynamic heart model based on averaged MRI data, MICCAI 2003, LNCS 2878, 549-555, 2003.

11. Fitzpatrick, J.M., West, J.B., Maurer, Jr. C.R., Predicting error in rigid-body point-based registration, IEEE Trans. Med. Imaging, 17:694-702, 1998. 